\title{
The Mediating Role of Negative Repetitive Thoughts in the Relationship between Brain Behavioral Systems and Insomnia Severity in Students
}

\author{
Ahmad Mansouri ${ }^{1, \text {, (D), Nasibe Mansouri }}{ }^{2}$, Hadi Bagheri ${ }^{3}$
}

\footnotetext{
${ }^{1}$ Assistant Professor, Department of Psychology, Neyshabur Branch, Islamic Azad University, Neyshabur, Iran

${ }^{2} \mathrm{PhD}$ Student in Counseling, Department of Educational Psychology, Mashhad Branch, Islamic Azad University, Mashhad, Iran

${ }^{3}$ Assistant Professor, Department of Physical Education, Shahrood University of Technology, Shahrood, Iran

* Corresponding author: Ahmad Mansouri, Assistant Professor, Department of Psychology, Neyshabur Branch, Islamic Azad University, Neyshabur, Iran. E-mail: mansoury_am@yahoo.com
}

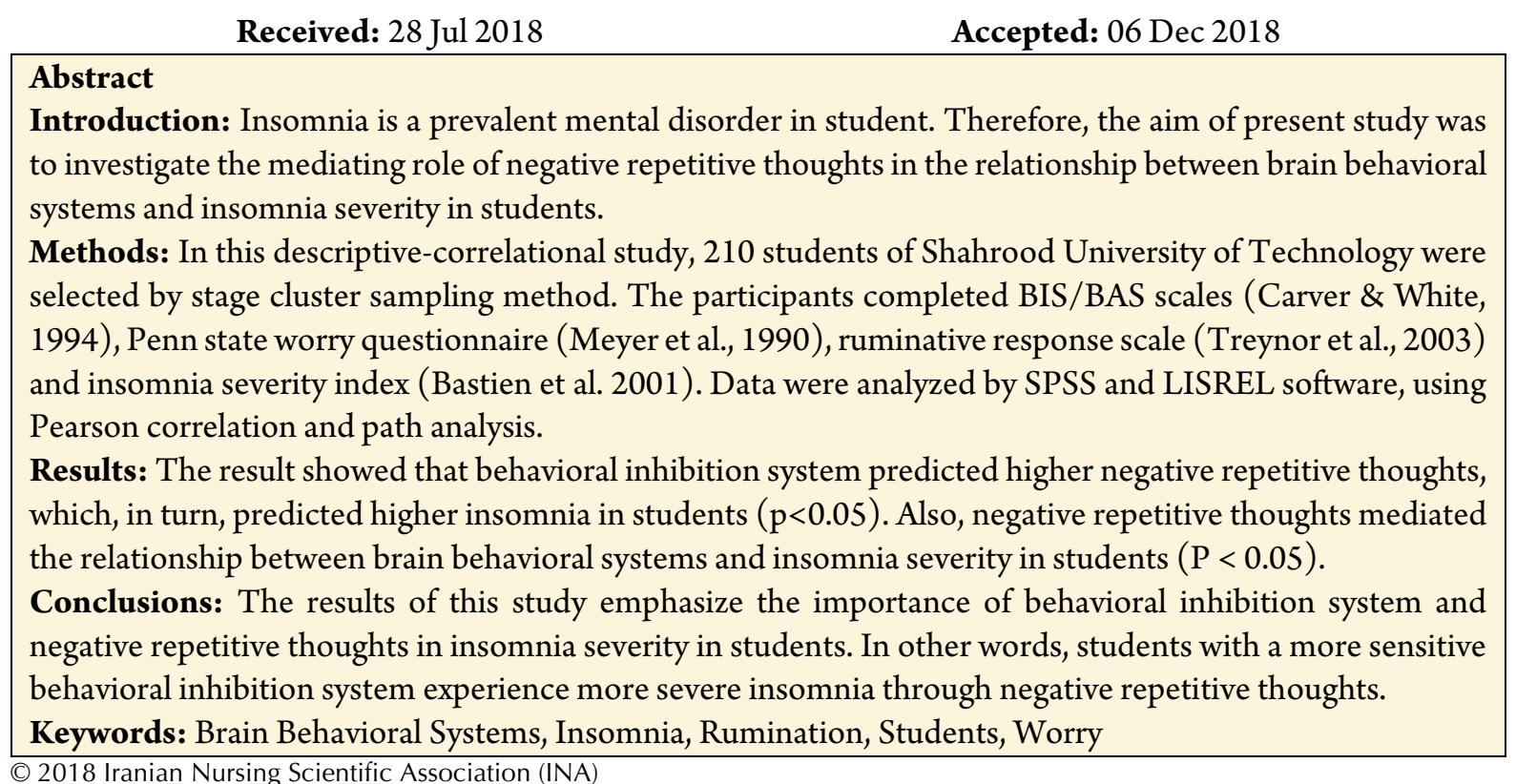




\title{
نقش ميانجى افكار تكرارشونده منفى در رابطه بين سيستمهاى مغزى رفتارى و شدت بى خوابى دانشجويان
}

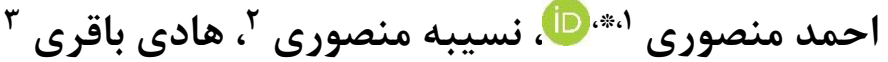

\begin{abstract}
' استاديار، كروه روانشناسى، واحد نيشابور، دانشكاه آزاد اسلامى، نيشابور، ايران

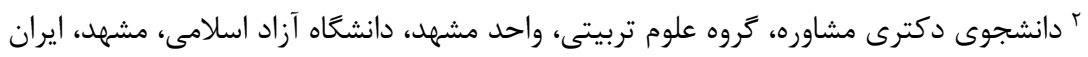

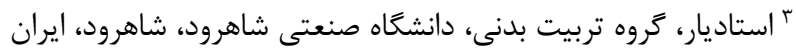

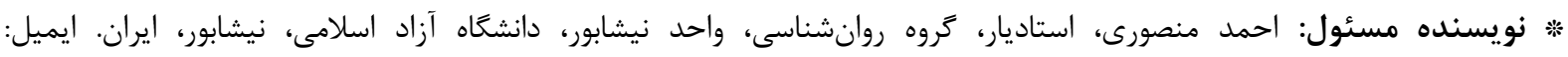
mansoury_am@yahoo.com

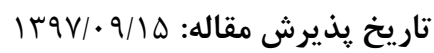

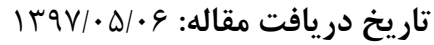

جكيده

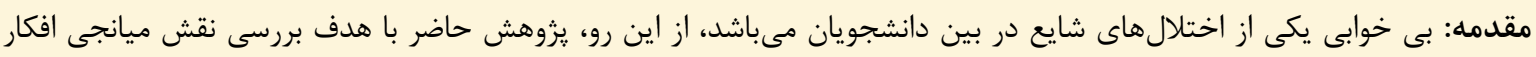

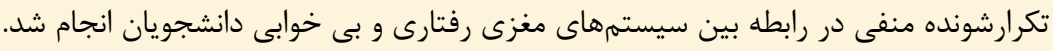

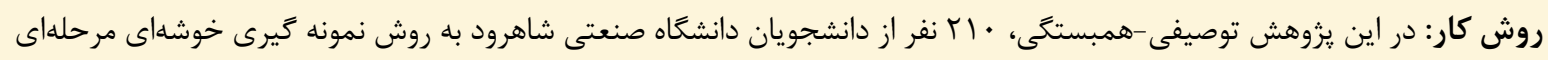

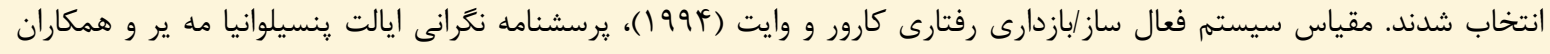

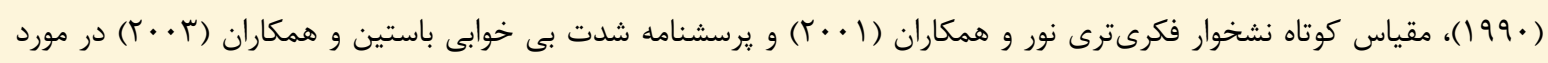

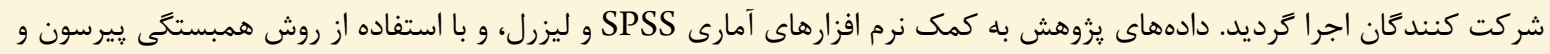
تحليل مسير تحليل شدند.

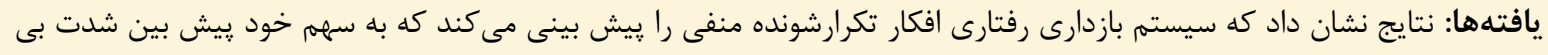

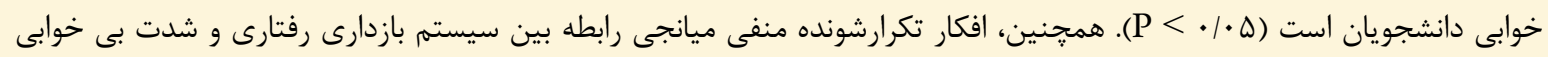

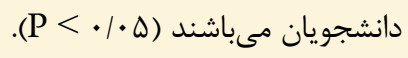

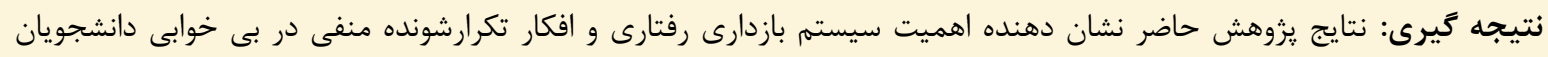

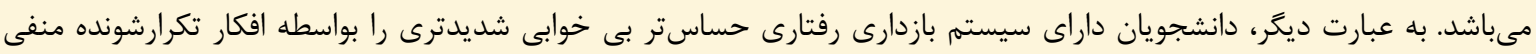

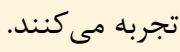

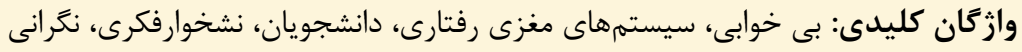

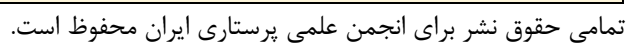

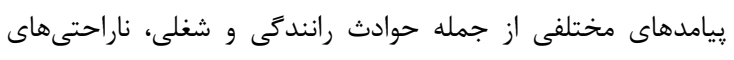

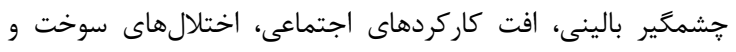

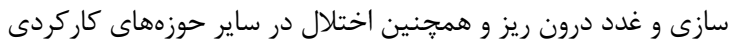

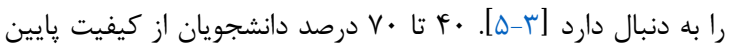

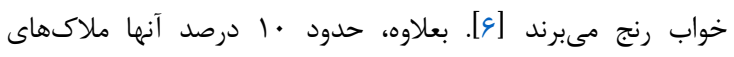

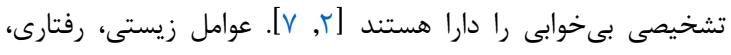

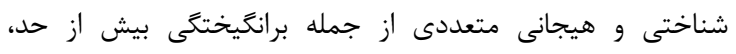

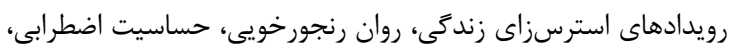

مقلدمه

خواب يكى از بخشهاى مهمم و ضرورى زندكى انسان است [1].

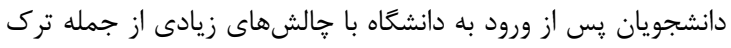

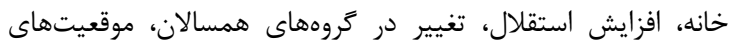

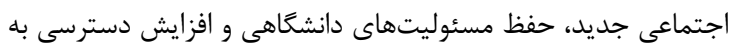

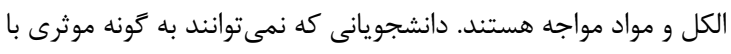

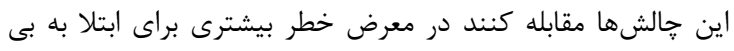

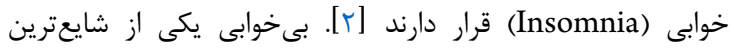

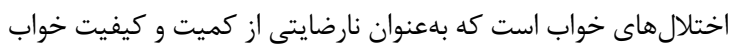

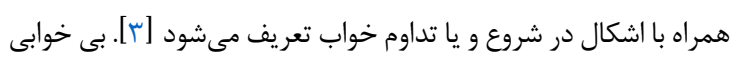


مدلها و الكَوهاى نشان مىدهد بين افكار تكرار شونده منفى نكَرانى و

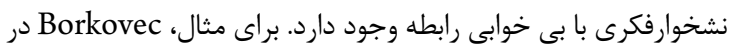

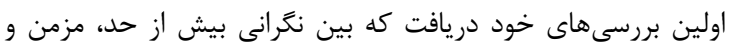

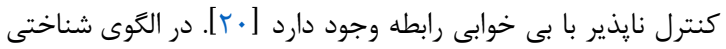

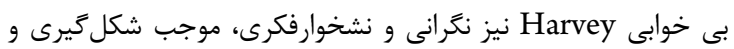

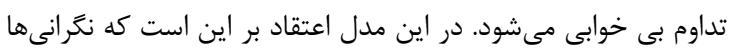

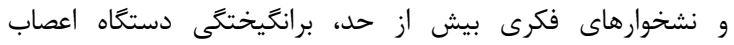

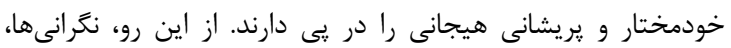

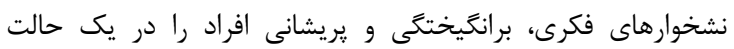

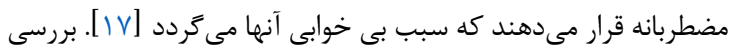

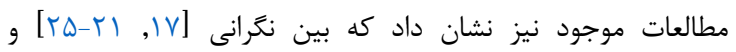

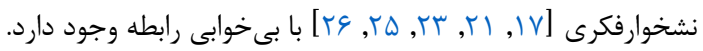

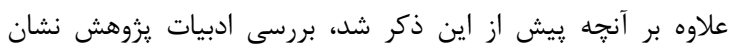

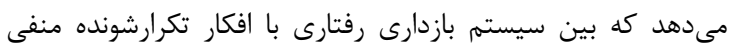

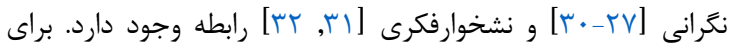

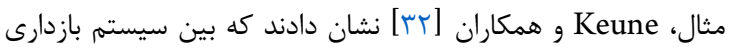

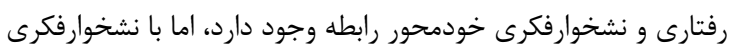

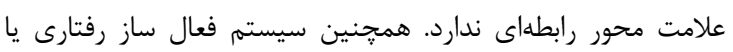

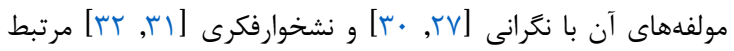

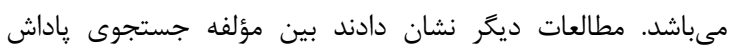

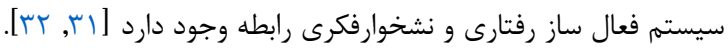

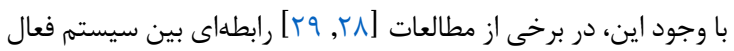

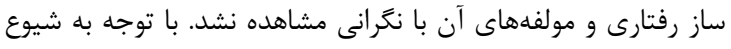

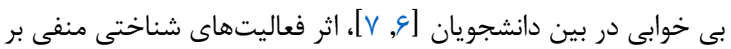

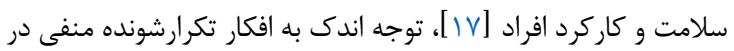

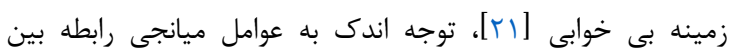

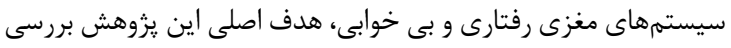

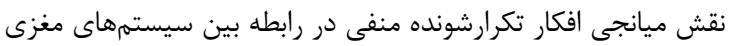
رفتارى و بى خوابى دانشجويان بود.

\section{روش كار}

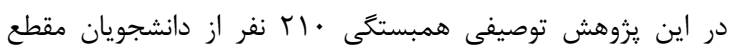

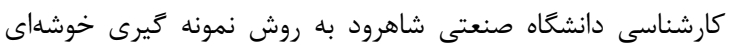

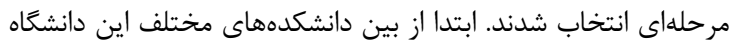

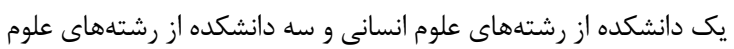

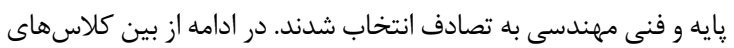

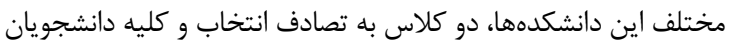

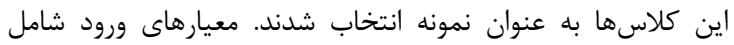

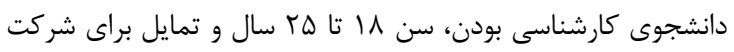

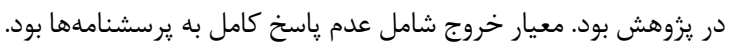

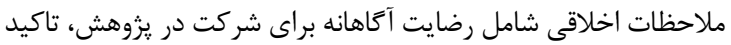

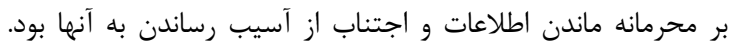

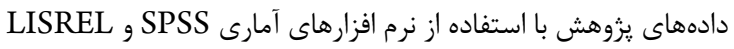

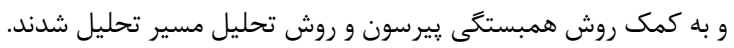

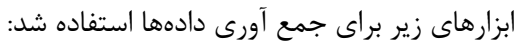

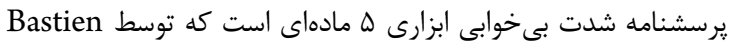

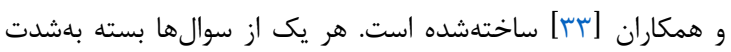

كمال كرايى، تمايل به درونى كردن مشكلات، ماندن زياد در رختخواب

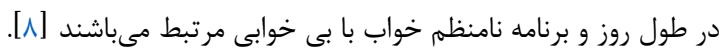

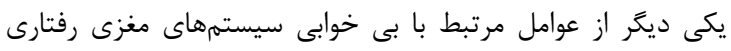
(Brain behavioral systems) اوليه خود دو سيستم مغزى رفتارى، يعنى سيستم كرايش و وبازدارى رفتارى (BAS: Behavioral Approach System) (BIS: Behavioral Inhibition System)

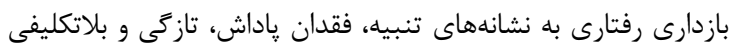

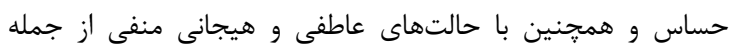

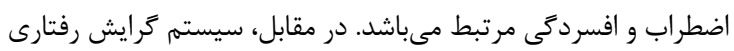

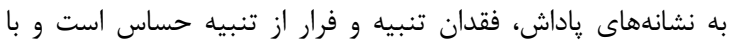

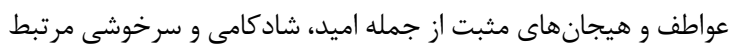

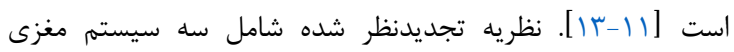

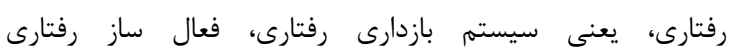
و جنكَ-كريز-انجماد (BAS: Behavioral Activation System) (FFFS: Fight-Flight-Freeze System)

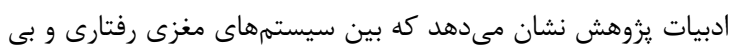

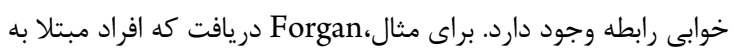

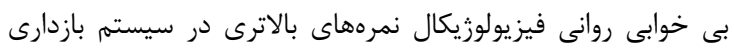

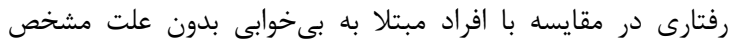

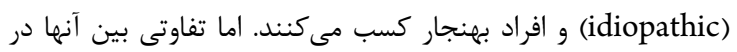

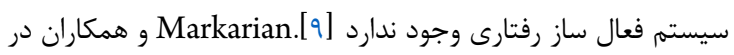

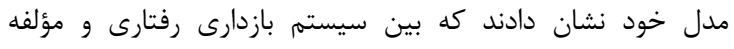

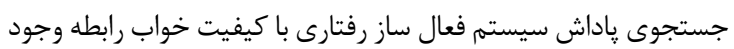

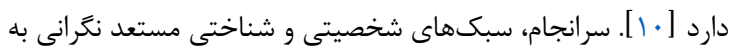

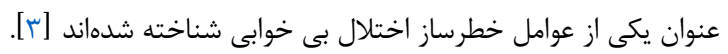

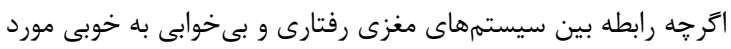

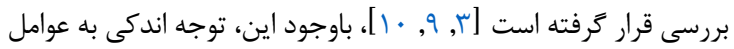

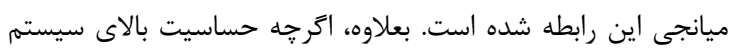

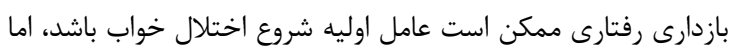

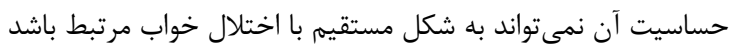

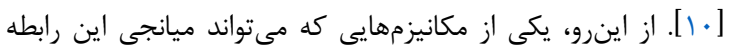
باشد، افكار تكرارشونده منفى (Negative repetitive thoughts)

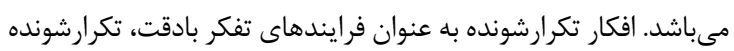

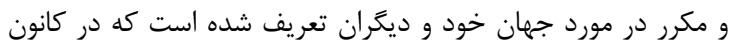

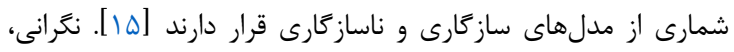

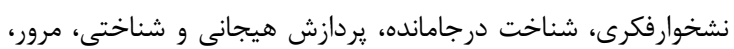

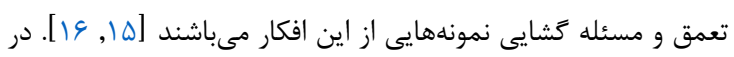

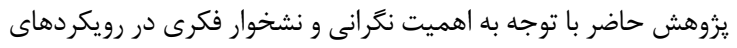

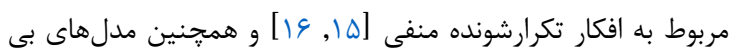

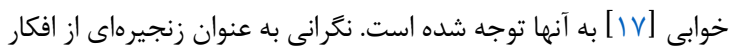

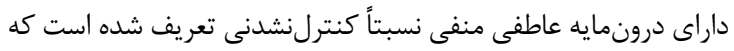

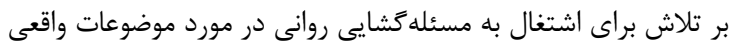

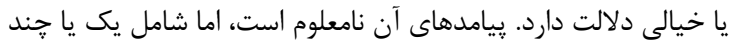

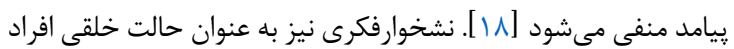

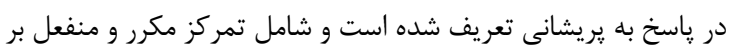

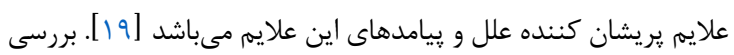


در يزوهش حاضر اعتبار آزمون به روش همسانى درونى 9 ه/ • بود. نتايج

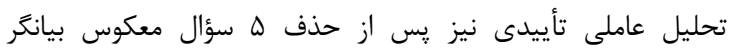

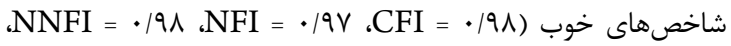

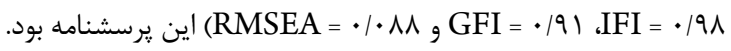

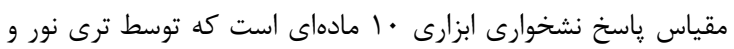

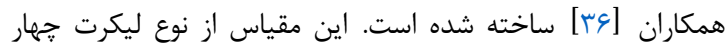

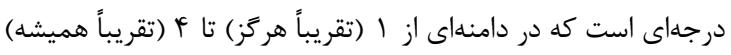

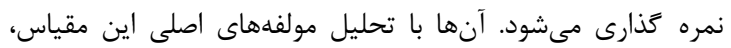

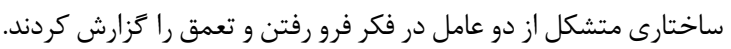

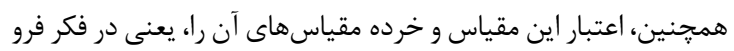

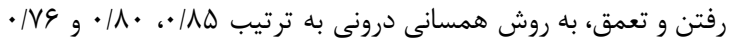

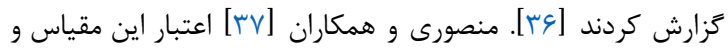

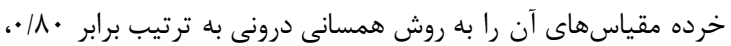

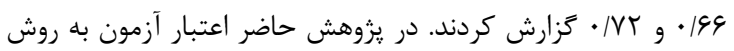

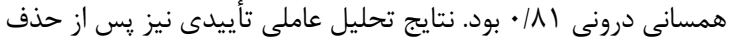

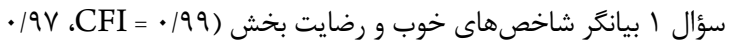

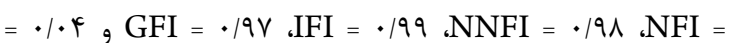

RMSEA اين مقياس بود. RFI

يافتهها

ميانگين سنى شركت كنند

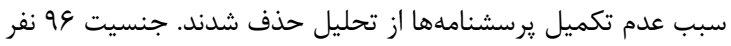

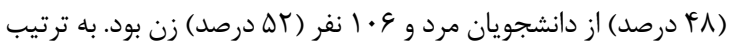
IN/D) IVV

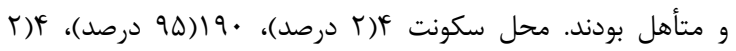

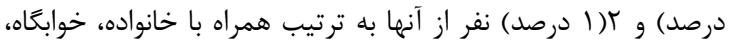

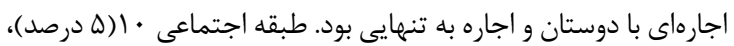

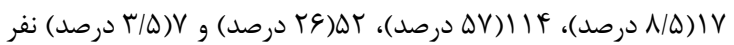

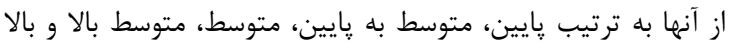

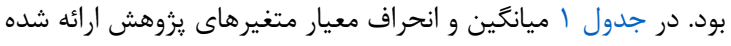

اختلال خواب موردنظر نمره • تأ أمى گيرند. اعتبار سازه آن بر اساس

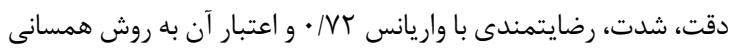

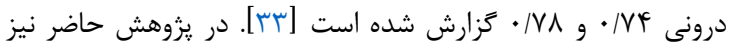

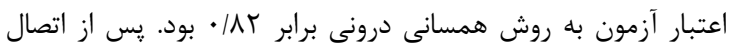

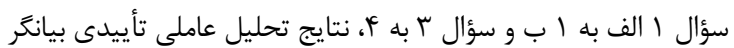

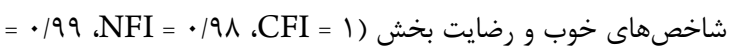

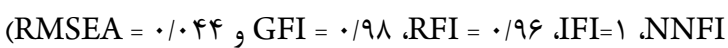
اين קرسشنامه بود.

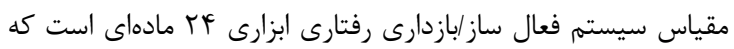

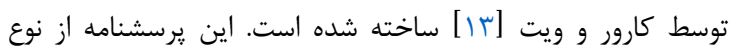

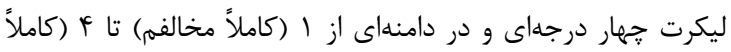

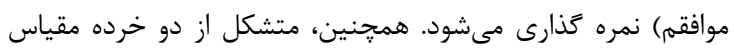

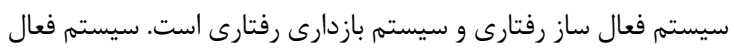

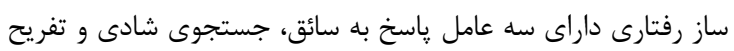

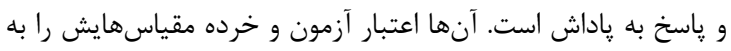

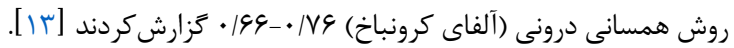

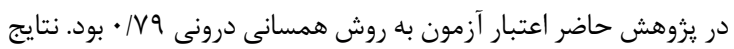

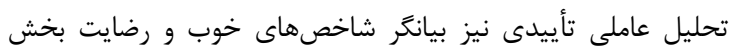

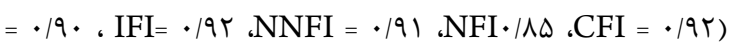
اين مقياس بود. براى اندازهخيرى افكار تكرارشونده منفى از دو يرسشنامه زير استفاده كرديد.

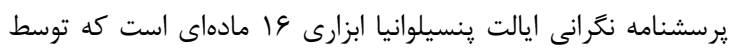

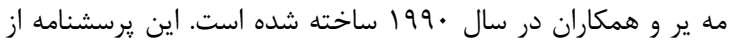

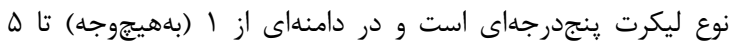

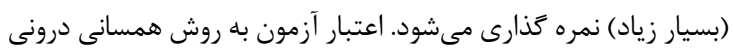

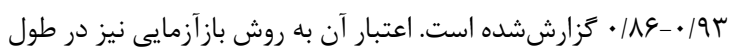

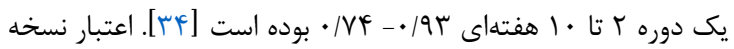

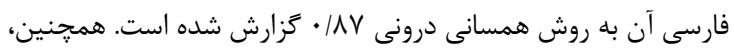

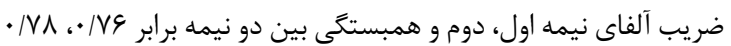

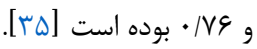

\begin{tabular}{|c|c|c|c|c|c|c|c|c|c|}
\hline \multirow[t]{9}{*}{9} & $\wedge$ & v & 9 & $\Delta$ & f & $r$ & $\frac{1}{r}$ & مربوط به متغ & متغول ا: شاخصهاى توصيفى و ضرايب هـ \\
\hline & & & & & & & & - & سيستم بازدارى رفتارى \\
\hline & & & & & & & - & $\cdot / r r \varphi^{*}$ & سيستم فعال ساز رفتارى \\
\hline & & & & & & - & $\cdot / V 9 Y^{* * *}$ & $\cdot / T Y Y^{* * a}$ & سائق - سيستم فعال ساز \\
\hline & & & & & - & $\cdot / r V^{m *}$ & $\cdot 19 \Delta V^{* m}$ & $\cdot / T r D^{* * m}$ & جستجوى شادى - سيستم فعال ساز \\
\hline & & & & - & $\cdot / \Upsilon \wedge \uparrow^{\mathrm{F} * m}$ & $\cdot / \boldsymbol{r} \cdot r^{* \ldots * 0}$ & $\cdot \mid \Lambda \cdot 1^{* * * a}$ & 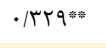 & ياسخ به هاداش - سيستم فعال ساز \\
\hline & & & - & $\cdot \mu r$ & $\cdot / \cdot 11$ & $-\cdot 1 \cdot 19$ & $\cdot / \cdot r \Lambda$ & $\cdot / T V Y^{* m^{*}}$ & نغرانى \\
\hline & & - & $\cdot \mid \Delta \Lambda \uparrow^{* * *}$ & $-\cdot / \cdot \wedge r$ & $\cdot 1 \cdot 19$ & $-\cdot / \cdot 91$ & $-\cdot / \cdot \Delta \cdot$ & $\cdot / 1 f^{*}$ & نشخوارفكرى \\
\hline & - & $\cdot / \Lambda \cdot V^{*=}$ & $\cdot / 9011^{* *}$ &.$- / \cdot r q$ & $.1 \cdot 10$ & $-.1 .+9$ & $\cdot / \cdot \cdot 1$ & $\cdot / r T Y^{* a n}$ & افكار تكرارشونده منفى \\
\hline- & 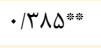 & 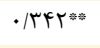 & $\cdot / r \Delta^{* * *}$ & $-\cdot / 1 \mathrm{rV}$ &.$/ \cdot 11$ &.$- / 1 \% 9$ & $-\cdot / 1 \cdot 1$ & $\cdot / \mid \Delta \Lambda^{*}$ & بى خوابى \\
\hline $11 / 9$. & $F q / \Delta V$ & $19 / \cdot V$ & $r \cdot / \Delta \cdot$ & $I V / I \Delta$ & 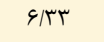 & $\mid r / \cdot 9$ & $r \Delta / \varphi \mid$ & $|\Delta / 1|$ & ميانكين \\
\hline$\Delta / 9 T$ & $F r / 90$ & $\Delta / V I$ & $1 \cdot / A V$ & $r / \Delta \Delta$ & $1 / \pi 4$ & 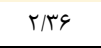 & F/gT & $r|\Delta|$ & انحراف استاندارد \\
\hline
\end{tabular}

مقياسهاى آن يعنى سائق، پاسخ به پاداش و جستجوى شادى با افكار

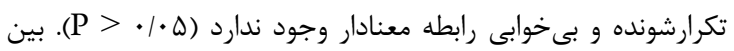

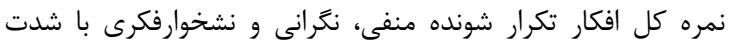

نتايج جدول ا نشان مىدهد كه بين سيستم بازدارى رفتارى با نمره

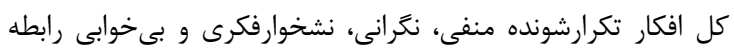

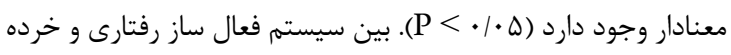


NNFI: Non-Normed (Normed fit index

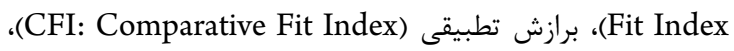
برازش افزايشى (Incremental Fit IFI: Index) و تقريب ريشه RMSEA: Root Mean Square Error of ) ميانگين مجذورات فرايشى)

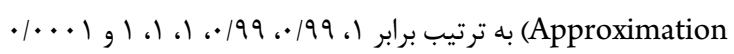

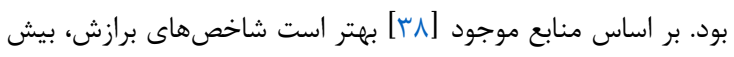

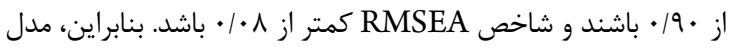
نهايى يزوهش حاضر برازش مطلوب و مناسبى دارد (تصوير ()).
بى خوابى رابطه وجود دارد (ه • • > P). علاوه بر اين، بررسى نرمال

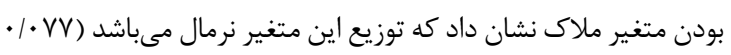

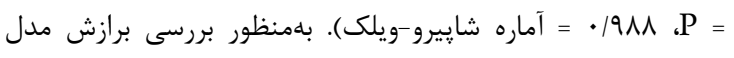

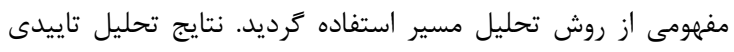

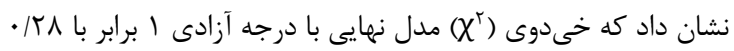

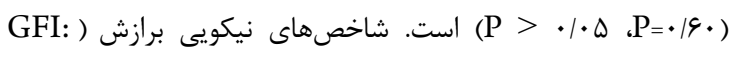
Adjusted (Goodness of Fit Index NFI: (AGFI: Goodness of Fit Index

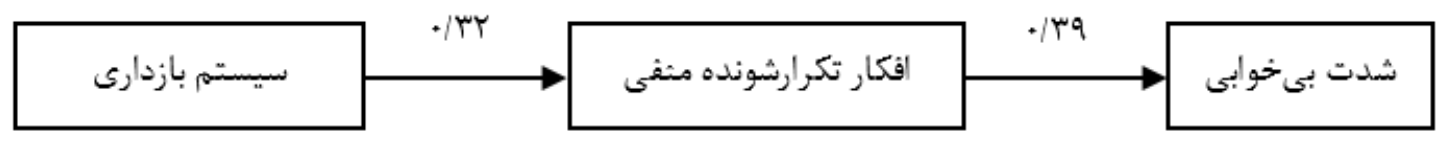

تصوير ا: مدل برازش شده مربوط به رابطه بين سيستمهاى مغزى رفتارى، افكار تكرارشونده منفى و شدت بى خوابى

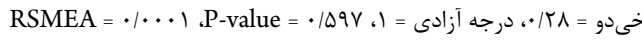

بنابراين، نتايج فوق بيشنهاد مى كند دانشجويانى كه سيستم بازدارى

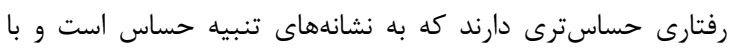

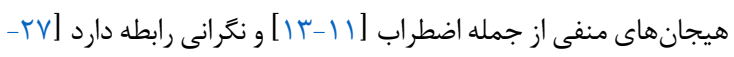

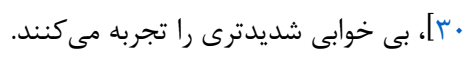

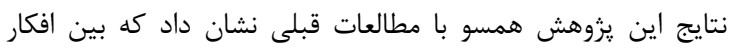

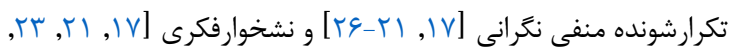

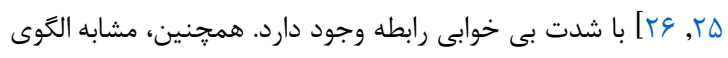

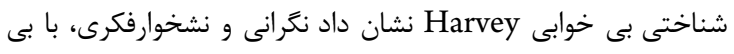

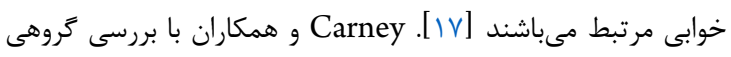

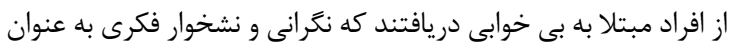

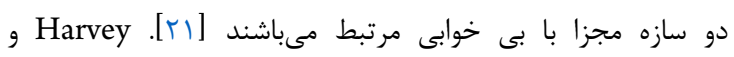
Greenall

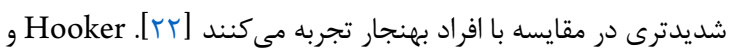

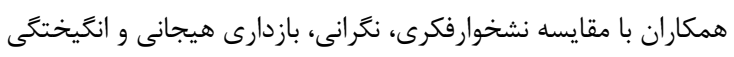

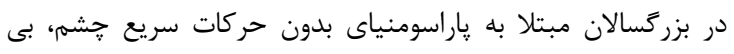

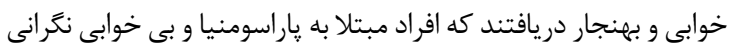

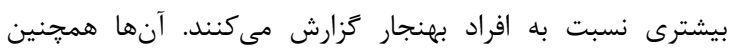

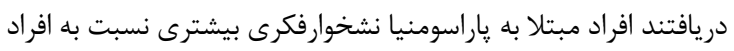

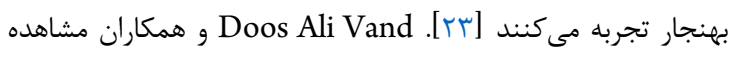

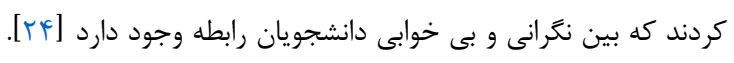
Mitchel

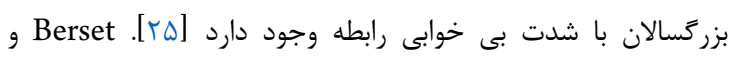

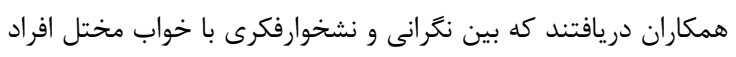

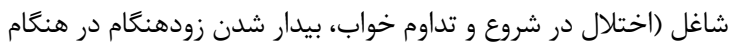

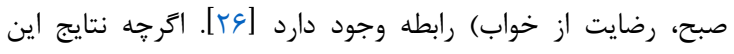

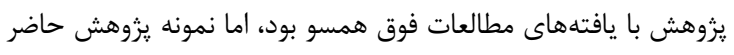

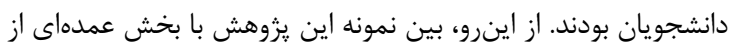

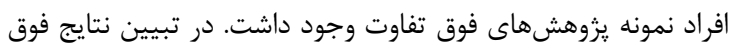

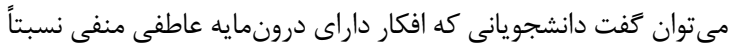

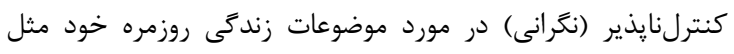

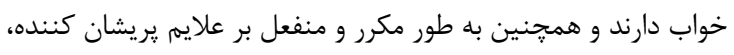

نتايج تصوير ا نشان مى دهد كه سيسته بازدارى رفتارى بيشبين افكار

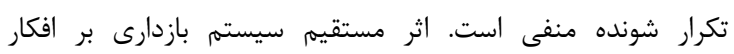

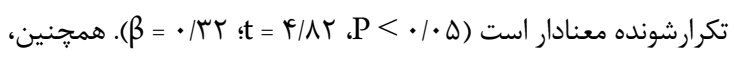

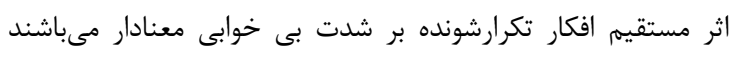

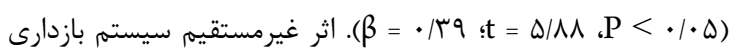

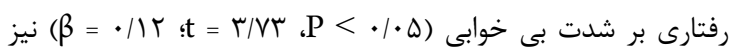

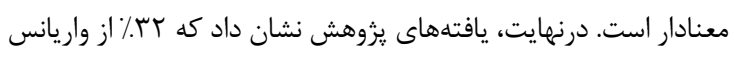

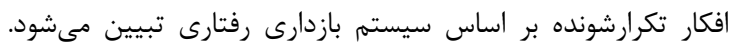

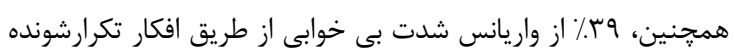
قابل تبيين است.

يروهش حاضر با هدف بررسى نقش ميانجى افكار تكرارشونده منفى در

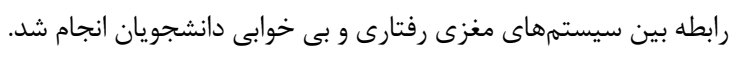

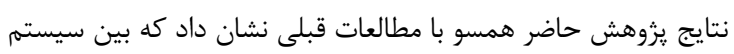

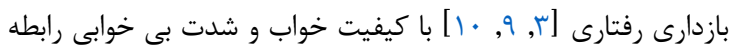

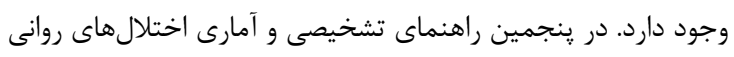

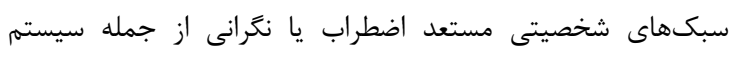

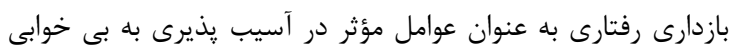
مطرح شده است [ץ]. Forgan با مقايسه حساسيت سيستم بازدارى و و

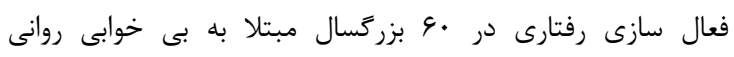

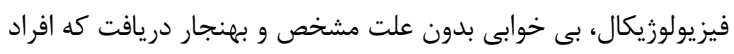

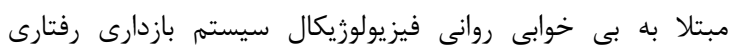

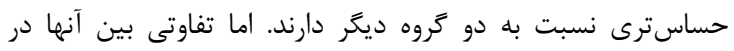

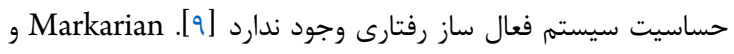

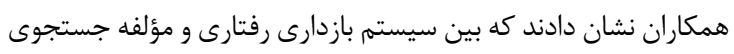

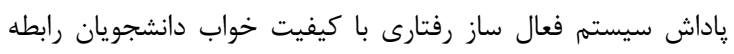

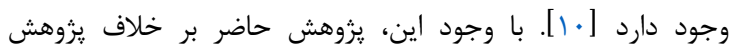
[7 Forgan اخير [•1] به بررسى نقش ميانجى افكار تكرار شونده در رابطه بين إندان

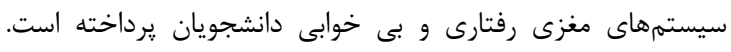




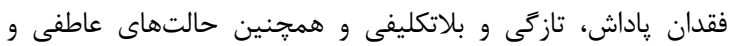

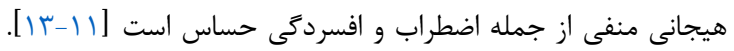

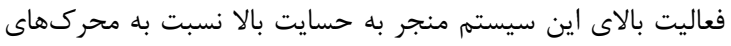

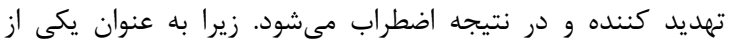

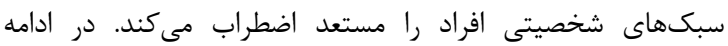

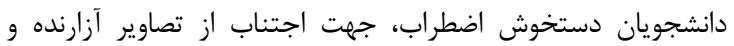

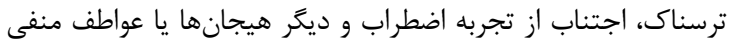

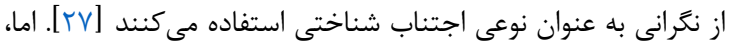

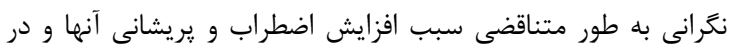

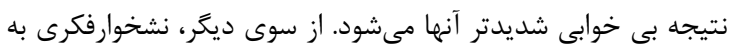

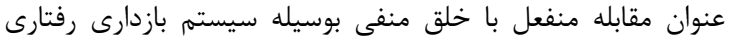

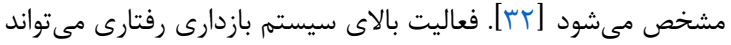

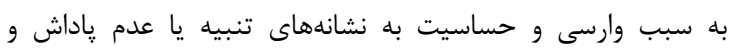

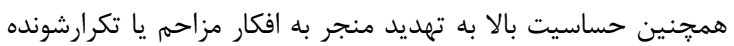

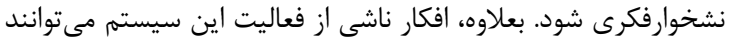

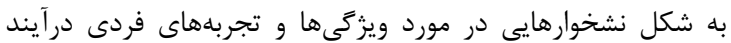

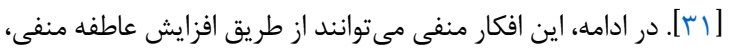

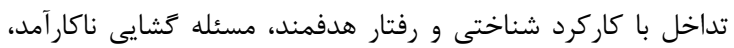

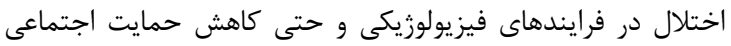

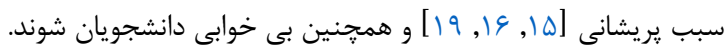

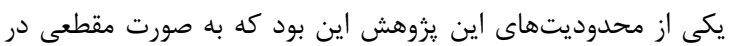

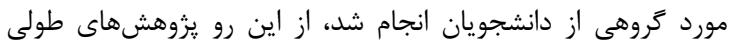

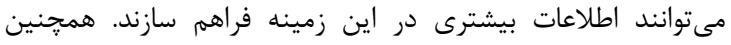

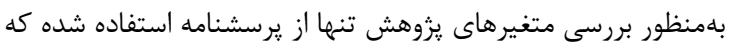

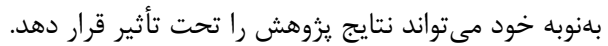

\section{نتيجه تيرى}

نتايج يُوهش حاضر نشان داد كه افكار تكرارشونده منفى ميانجى رابطه

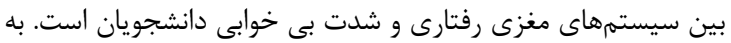

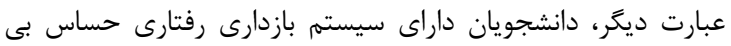

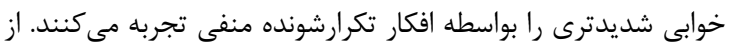

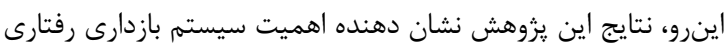

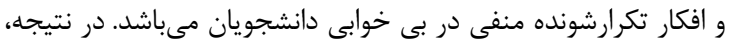

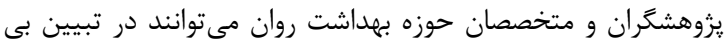

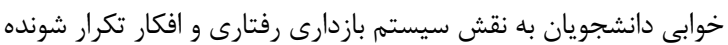
منفى توجه داشته باشند.

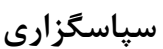

از همكارى تمامى دانشجويان و ساير افرادى كه در انجام اين يزوهش

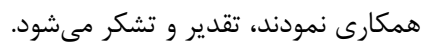

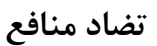

هيجكَونه تضاد منافعى براى نويسندكان اين مقاله وجود ندارد.

\section{References}

1. Hiller RM, Johnston A, Dohnt H, Lovato N, Gradisar M. Assessing cognitive processes related to insomnia: A review and measurement guide for Harvey's cognitive model for the maintenance of insomnia. Sleep Med Rev.
علل و ييامدهاى علايم خود تمركز دارند (نشخوارفكرى)، بى خوابى

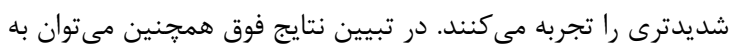

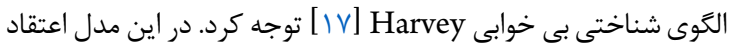

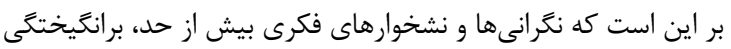

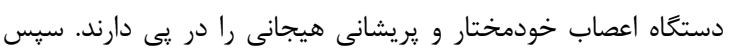

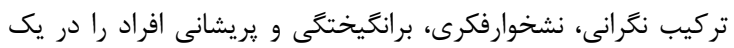

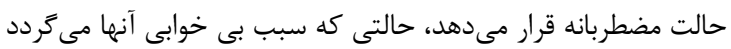

نتايج اين يزوهش همجنين همسو با مطالعات قبلى نشان داد كه بين

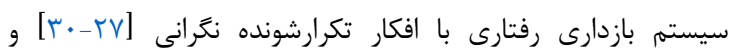

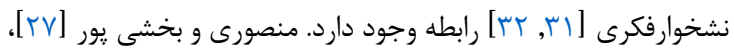

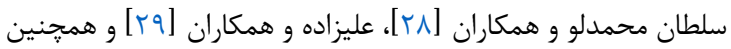

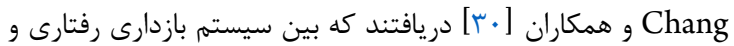

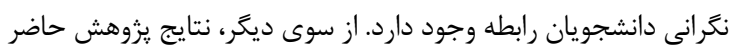

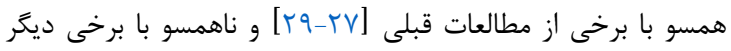

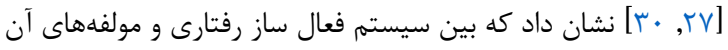

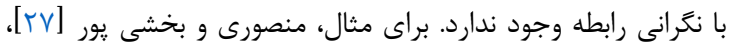

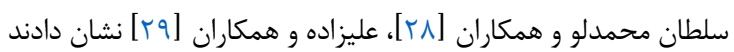

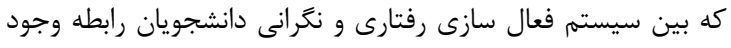

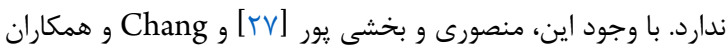

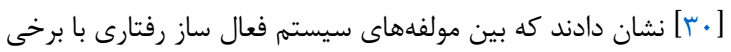

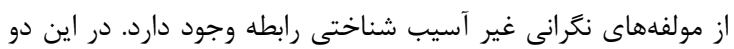

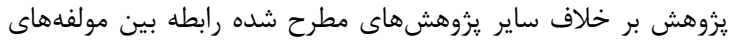

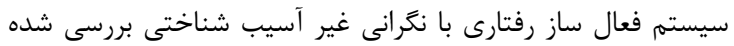

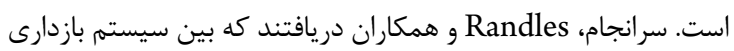

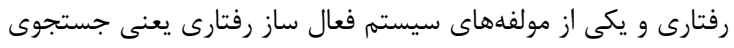

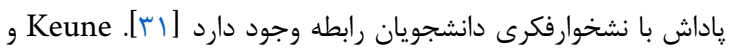

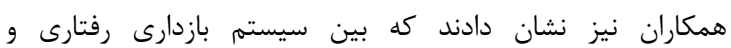

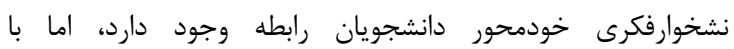
نشخوارفكرى علامت محور رابطهاى ندارد. بعلاوه، رابطهاى بين بين

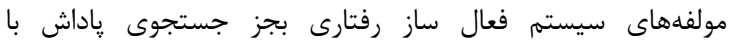
نشخوارفكرى وجود ندارد [بr].

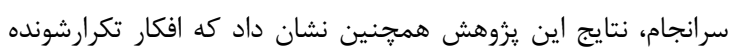

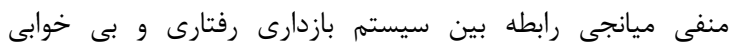

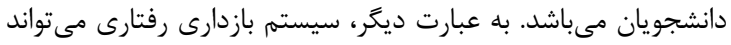

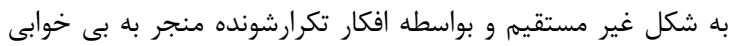

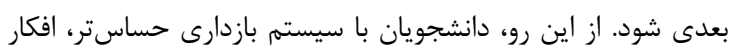

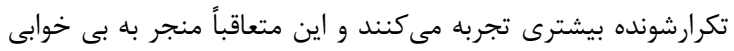

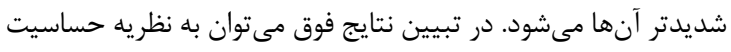

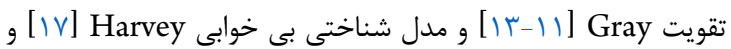

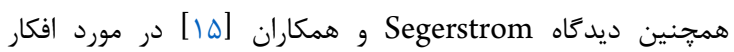

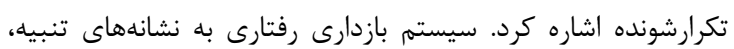

2015;23:46-53. doi: 10.1016/j.smrv.2014.11.006 pmid: 25645129

2. Taylor DJ, Gardner CE, Bramoweth AD, Williams JM, Roane BM, Grieser EA, et al. Insomnia and mental health 
in college students. Behav Sleep Med. 2011;9(2):107-16. doi: 10.1080/15402002.2011.557992 pmid: 21491233

3. American Psychiatric Association. Diagnostic and statistical manual of mental disorders (DSM- $\left.5^{\circledast}\right)$. 5rd, editor. Washington DC: APA; 2013.

4. Alidostim M, Hemati Z. Relationship between quality of sleep and quality of life in dialysis patients of dialysis centers in Chaharmahal and Bakhtiari, Iran. J Health Syst Res. 2012;8(3):515-21.

5. Ttaboli $\mathrm{H}$, Shabani N. An investigation of the relationship between individual preparedness of workers in crisis management and their overall health and sleep quality. $\mathrm{J}$ Health Syst Res. 2017;12(4):472-7.

6. Gellis LA, Park A, Stotsky MT, Taylor DJ. Associations between sleep hygiene and insomnia severity in college students: cross-sectional and prospective analyses. Behav Ther 2014;45(6):806-16

doi: 10.1016/j.beth.2014.05.002 pmid: 25311289

7. Taylor DJ, Bramoweth AD, Grieser EA, Tatum JI, Roane BM. Epidemiology of insomnia in college students: relationship with mental health, quality of life, and substance use difficulties. Behav Ther. 2013;44(3):33948. doi: 10.1016/j.beth.2012.12.001 pmid: 23768662

8. Morin CM, Drake CL, Harvey AG, Krystal AD, Manber $\mathrm{R}$, Riemann D, et al. Insomnia disorder. Nat Rev Dis Primers. 2015;1:15026. doi: 10.1038/nrdp.2015.26 pmid: 27189779

9. Forgan G. Psychophysiological insomnia and idiopathic insomnia: The role of self-regulatory behaviour systems. England, Glasgow: University of Glasgow; 2010.

10. Markarian SA, Pickett SM, Deveson DF, Kanona BB. A model of BIS/BAS sensitivity, emotion regulation difficulties, and depression, anxiety, and stress symptoms in relation to sleep quality. Psychiatry Res. 2013;210(1):281-6. doi: 10.1016/j.psychres.2013.06.004 pmid: 23845417

11. Gray JA. The psychophysiological basis of introversionextraversion. Behav Res Ther. 1970;8(3):249-66. pmid: 5470377

12. Gray J. The neuropsychology of anxiety: an enquiry into the functions of the septo-hippocampal system. Oxford Psychology. England: Oxford University Press; 1982.

13. Carver CS, White TL. Behavioral inhibition, behavioral activation, and affective responses to impending reward and punishment: the BIS/BAS scales. J Pers Soc Psychol. 1994;67(2):319.

14. Gray J, McNaughton N. The neuropsychology of anxiety: An enquiry into the functions of the septo-hippocampal system. Oxford: Oxford University Press; 2003.

15. Segerstrom SC, Stanton AL, Alden LE, Shortridge BE. A multidimensional structure for repetitive thought: what's on your mind, and how, and how much? J Pers Soc Psychol. 2003;85(5):909-21. doi: 10.1037/00223514.85.5.909 pmid: 14599253

16. Watkins ER. Constructive and unconstructive repetitive thought. Psychol Bull. 2008;134(2):163-206. doi: 10.1037/0033-2909.134.2.163 pmid: 18298268

17. Harvey AG. A cognitive model of insomnia. Behav Res Ther. 2002;40(8):869-93. pmid: 12186352

18. Borkovec TD, Robinson E, Pruzinsky T, DePree JA. Preliminary exploration of worry: some characteristics and processes. Behav Res Ther. 1983;21(1):9-16. pmid: 6830571
19. Nolen-Hoeksema S, Wisco BE, Lyubomirsky S. Rethinking Rumination. Perspect Psychol Sci. 2008;3(5):400-24. doi: 10.1111/j.17456924.2008.00088.x pmid: 26158958

20. Borkovec T. Pseudo (experiental)-insomnia and idiopathic (objective) insomnia: theoretical and therapeutic issues. Adv Behav Res Ther 1979;2(1):27-55.

21. Carney CE, Harris AL, Moss TG, Edinger JD. Distinguishing rumination from worry in clinical insomnia. Behav Res Ther. 2010;48(6):540-6. doi: 10.1016/j.brat.2010.03.004 pmid: 20362977

22. Harvey AG. Beliefs about the utility of presleep worry: An investigation of individuals with insomnia and good sleepers. Cogn Ther Res. 2003;27(4):403-14. doi: 10.1023/A: 1025420429735

23. Hooker KE. A controlled comparative investigation of rumination, worry, emotional inhibition and arousability in adults with nREM parasomnias, insomnia and good sleepers. Scotland: University of Glasgow; 2011.

24. Doos Ali Vand H, Gharraee B, Farid AA, Bandi MG. Prediction of insomnia severity based on cognitive, metacognitive, and emotional variables in college students. Explore (NY). 2014;10(4):233-40. doi: 10.1016/j.explore.2014.04.005 pmid: 25037666

25. Mitchell L, Mogg K, Bradley BP. Relationships between insomnia, negative emotionality and attention control. Sleep Biol Rhythms. 2012;10(3):237-43. doi: 10.1111/j.1479-8425.2012.00567.x

26. Berset M, Elfering A, Luthy S, Luthi S, Semmer NK. Work stressors and impaired sleep: rumination as a mediator. Stress Health. 2011;27(2):e71-82. pmid: 27486625

27. Mansouri A, Bakhshipur A. The relationship between behavioral activation and inhibition systems on the pathological and non-pathological worry. J Babol Univ Med Sci. 2010;12(1):59-64.

28. Soltan Mohammadlou S, Gharraee B, Fathali Lvasani F, Gohari M. The relationship of Behavioral Activation and Inhibition Systems (BAS/BIS), difficulty of emotional regulation, metacognition with worry. Res Cogn Behav Sci. 2013;3(2):85-100.

29. Alizadeh A, Hasanzadeh L, Mahmood Aliloo M, Poursharifi H. Predict of worry based on behavioral activation and inhibition systems(BAS/BIS), cognitive emotion regulation and intolerance of uncertainty in students. Iran J Cardiovasc Pract. 2014;2(3):1-11.

30. Chang EC, Zumberg KM, Sanna LJ, Girz LP, Kade AM, Shair SR, et al. Relationship between perfectionism and domains of worry in a college student population: Considering the role of BIS/BAS motives. Pers Individ Differ 2007;43(4):925-36. doi: 10.1016/j.paid.2007.02.026

31. Randles D, Flett GL, Nash KA, McGregor ID, Hewitt PL. Dimensions of perfectionism, behavioral inhibition, and rumination. Pers Individ Differ. 2010;49(2):83-7. doi: 10.1016/j.paid.2010.03.002

32. Keune PM, Bostanov V, Kotchoubey B, Hautzinger M. Mindfulness versus rumination and behavioral inhibition: A perspective from research on frontal brain asymmetry. Pers Individ Differ. 2012;53(3):323-8. doi: 10.1016/j.paid.2012.03.034

33. Bastien $\mathrm{CH}$, Vallieres A, Morin CM. Validation of the Insomnia Severity Index as an outcome measure for insomnia research. Sleep Med. 2001;2(4):297-307. pmid: 11438246 
34. Meyer TJ, Miller ML, Metzger RL, Borkovec TD. Development and validation of the Penn State Worry Questionnaire. Behav Res Ther. 1990;28(6):487-95. pmid: 2076086

35. Mansouri A, Khodayarifard M, Besharat M, Gholamali Lavasani M. Moderating and Mediating role of spiritual coping and cognitive emotion regulation Strategies in the relationship between transdiagnostic factors and symptoms of generalized anxiety disorder: Developing a conceptual model. J Res Behav Sci. 2018;16(2):130-42.
36. Treynor W, Gonzalez R, Nolen-Hoeksema S. Rumination reconsidered: A psychometric analysis. Cogn Ther Res. 2003;27(3):247-59. doi: 10.1023/A:1023910315561

37. Mansouri A, Mansouri M, Mansouri N. The relation between schizotypay and depression symptom according to mediator role of rumination. CPAP. 2015;2(11):99108 .

38. Meyers LS, Gamst G, Guarino AJ. Applied multivariate research: Design and interpretation. London: Sage publications; 2006. 